\title{
China y el arte de la guerra (tecnológica)
}

\author{
Sergio Cesarin y \\ Gabriel Balbo
}

\author{
scesarin@untref.edu.ar \\ CONICET \\ gbalbo@unaj.edu.ar \\ Universidad Nacional Arturo Jauretche
}

Recibido: $7 / 10 / 2020$.

Aceptado: $7 / 12 / 2020$

Resumen: China se encamina a convertirse en una potencia tecnológica a mediados del siglo $X X I$. Este proceso se funda sobre cuatro pilares: sostenido incremento del presupuesto asignado al desarrollo científico-tecnológico, su directa vinculación con estrategias de innovación a fin de aumentar la productividad y sostener competitividad exportadora, creación e internacionalización de firmas tecnológicas, y expectativas gubernamentales por cerrar las brechas de conocimiento aún existentes que separan a China de competidores asiáticos y occidentales. A lo largo de cuatro décadas, las reformas pro mercado confirman esta senda de transformación y generación de capacidades en sectores tecnológicos de punta. La competencia estratégica con Estados Unidos acelera esta carrera y confirma objetivos sobre posicionamiento de firmas tecnológicas chinas como líderes en inteligencia artificial (IA), telecomunicaciones y redes $5 \mathrm{G}$.

Palabras clave: ciencia; alianzas; poder tecnológico; empresas; innovación; geopolítica

* Cómo citar este artículo: Cesarin, S. y Balbo, G. (2020). China y el arte de la guerra (tecnológica). Relaciones Internacionales, 29(59), 110.

https://doi.org/10.24215/23142766e110

Editor: Juan Alberto Rial, Instituto de Relaciones Internacionales Facultad de Ciencias Jurídicas y Sociales (Universidad Nacional de La Plata)
Entidad editora: Relaciones

Internacionales, es una publicación del Instituto de Relaciones Internacionales (Facultad de Ciencias Jurídicas y Sociales (Universidad Nacional de La Plata Argentina) Vol 29 - no 59/2020

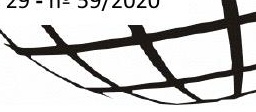




\title{
China and the art of (technological) war
}

\section{Sergio Cesarin1 y Gabriel Balbo2}

\begin{abstract}
China is on the way to become a technological power in the mid-21st Century. This process is based on four pillars: sustained increase in science and technology development budget allocations, its direct link to innovation strategies to increase productivity and upkeep exploration competitiveness, high-tech firm creation and internationalization, and national goals aimed at closing the gap between domestic S\&T capacities and those of the more advanced developed Asian and Western economies. Over four decades, pro-market reforms have confirmed this path, towards transformation and capacity building in cutting edge technological sectors. Strategic tensions with the United States speed up this race and confirm China's national goals for its companies to take the lead in artificial intelligence (AI), telecommunications and $5 \mathrm{G}$ networks.
\end{abstract}

Keywords: science; alliances; technological power; business; innovation; geopolitics

1 Investigador del CONICET, Docente de la Especialización en estudios chinos del IRI, y Coordinador del Centro de estudios sobre Asia del Pacífico e India (CEAPI) de la UNTREf.

2 Docente-investigador de la UNAJ y UNLP, Coordinador del Programa Radar VTIC UNAJ, de Vigilancia Tecnológica e Inteligencia Competitiva. 
“...Sun Zi dice: las diferentes maneras de combatir por el fuego se reducen a cinco. La primera consiste en quemar a los hombres; la segunda, en quemar las provisiones; la tercera, en quemar los bagajes; la cuarta, en quemar los arsenales y almacenes; y la quinta, en utilizar proyectiles incendiarios..." Sun Zi, El arte de la guerra, S V a.C

\section{Introducción}

La máxima de Sun Zi no suena extraña en tanto proviene de una cultura-civilización caracterizada por la inventiva e innovación desde centurias antes de la era cristiana. Sus logros científicos y técnicos fueron aplicados al desarrollo económico-social del Imperio como también al arte de la guerra en diferentes campos, como la astronomía, navegación marítima, cálculo, diseño arquitectónico, sistemas de irrigación o metalurgia. Un derrotero marcado por conquistas técnicas permitieron la supervivencia del Imperio del Centro por más de dos milenios. En este tiempo, y como ayer, la historia vuelve a repetirse bajo diferentes matices. Por ello, en los albores de la tercera década del siglo XXI, China persiste en lograr metas de mediano y largo plazo tendientes a incrementar sus capacidades en el plano tecnológico y vincularlas con mayores dosis de poder económico, financiero, comercial y militar.

Sobre la base de una "visión estratégica", el proceso de transformación estructural en el plano tecnológico evoluciona sobre dos ejes interactuantes: en primer lugar, continuar el sendero trazado por los reformistas chinos a fines del siglo XX quienes consideraron la modernización tecnológica como uno de los cuatro pilares imprescindibles para revertir el subdesarrollo nacional; los líderes de entonces -con Deng Xiaoping a la cabeza- asumieron este desafío, determinante para que China recuperara posiciones perdidas de poder. Los estrategas chinos de aquel momento consideraron inescindibles la apertura económica pro mercado, la generación de un favorable ambiente económico apto para la recepción de inversiones externas (ie), la formación de recursos humanos y la transferencia tecnológica por parte de firmas extranjeras instaladas en enclaves económicos o zonas económicas especiales (Z.E.E), con el último fin de generar capacidades endógenas mediante técnicas de aprendizaje y asimilación de conocimientos (Naughton, 1996).

Un segundo eje fue la participación del capital extranjero asociado al nacional: capital y empresas extranjeras funcionaron como facilitadores en la búsqueda de nuevas capacidades científico-tecnológicas. El aporte combinado de socios extranjeros y financiamiento local (joint ventures) expandió internamente las fronteras y modalidades de "capitalismo schumpeteriano" creativo y emprendedor en una cultura con tradiciones mercantiles arraigadas, en una primera etapa, atraídos por bajos costos laborales y, en una segunda etapa, por oportunidades derivadas de una e-conomía de redes en expansión desde fines del pasado siglo. Este entorno nuevo político-institucional definido por la política de "puertas abiertas", en combinación con cambios generacionales, permitió luego el surgimiento de jóvenes emprendedores formados en su mayoría en instituciones académicas y centros de estudio de Estados Unidos, Europa, Japón, Australia, Singapur o Corea del Sur. 
Como resultado, durante las dos últimas décadas del siglo XX, China favoreció la incorporación de nuevas tecnologías acompañadas por medidas internas de promoción sectorial que dieron origen al modelo made in China sostenido por bajos costos laborales, industrias mano de obra intensivas y una economía de escala sin precedentes históricos. Una segunda etapa, desde inicios del siglo XXI, da lugar a la promoción de industrias capitalintensivas, surgimiento de start-ups y proyección global de firmas tecnológicas. Este pasaje signado por aumentos en los costos laborales, automatización y robotización productiva culminó la transición del modelo made in China al "designed in" China (Shenkar, 2005).

En la actualidad, el resultado de esta estrategia general es verificable en indicadores sobre inversiones estatales y privadas en desarrollo científico-tecnológico y fomento de la innovación -una ajustada simbiosis entre entorno institucional y creatividad moviliza el espíritu empresarial de millones de jóvenes ingenieros y técnicos formados en China y el extranjero. El cuadro mundial de capacidades científico-tecnológicas indica cómo empresas chinas (estatales y/o privadas) lideran mundialmente en registros sobre diseño, patentes y marcas (World Intelectual Property Organization, WIPO); asimismo, varias se ubican en la cima de los rankings sobre competitividad y proveedores confiables en el sector nuclear, energías no convencionales, aeroespacial, IA, redes $5 \mathrm{G}$ y plataformas de e commerce, entre otros.

A las puestas de la tercera década del siglo XXI, estos procesos han cristalizado un entorno global competitivo definido, principalmente, por la escalada de tensiones entre Estados Unidos y China. Los Estados Unidos han puesto en el centro de la puja estratégica con China la "guerra tecnológica". Según la National Defense Strategy (NDS, 2018), los Estados Unidos consideran a China un "competidor estratégico", una directa amenaza a su predominio global en tecnologías críticas, como TICs, telecomunicaciones e IA, capaz de intimidar naciones vecinas, alentar operaciones de ciberespionaje y-coaligadamente con Rusia e Irán- erosionar las bases del sistema político estadunidense. Como un sucedáneo de este conflicto bilateral, los planes de firmas chinas como Huawei (líder mundial en tecnologías $5 \mathrm{G}$ e IA) de instalar y operar redes en ALC agudizan mutuas desconfianzas e introducen nuevamente a nuestra región en un conflictivo escenario de tensiones sino-estadounidense, ya no sesgado por la ideología, sino por determinantes geopolíticos, pujas por influencia y determinismos de mercado.

En este contexto de escalada entre la "potencia declinante" y la "potencia emergente", analizar el proceso de transformación de China en potencia tecnológica, sus expectativas por liderar segmentos de última generación y fijar stándares globales, reviste particular interés por sus implicancias sistémicas pero, fundamentalmente, por sus impactos actuales y futuros en América Latina y el Caribe (ALC). La asociación con una China tecnológicamente avanzada genera debates en una región que aún basa la fortaleza de su sector externo en la exportación de commodities en desmedro de objetivos sobre desarrollos en C\&T3, aspecto que reafirma su inserción periférica en la economía mundial.

3 Katz, J. (2015) “La macro- y la microeconomía del crecimiento basado en los recursos naturales” en Bárcena, A. y Prado, A. (Eds.) Neoestructuralismo y corrientes heterodoxas en América Latina y el Caribe a inicios del Siglo XXI. CEPAL, Chile. 
Por todo lo expuesto, el presente trabajo analiza dos aspectos. El primero comprende factores y variables intervinientes en el proceso de transformación de China en potencia tecnológica. En segundo lugar, sus impactos en la disputa estratégica con Estados Unidos. Para el logro de estos propósitos, dividimos el trabajo en diferentes tópicos. Los tres primeros abordan la estrategia y trayectoria general de China dirigida al logro de avances en C\&T, la descripción enfatiza variables tales como el activo rol desempeñado por el Estado en la asignación de recursos aplicados a C\&T e I\&D, los sucesivos planes nacionales con metas definidas, la sostenibilidad de una estrategia con miras al largo plazo (mediados del siglo XXI) y sus derivaciones actuales, evidentes en indicadores sobre posicionamiento global de firmas tecnológicas chinas.

Un cuarto apartado analiza específicamente el Plan "Hecho en China 2025" como eje de la puja que sostiene con Estados Unidos en la carrera por el predomino tecnológico mundial. Finalmente, un último punto analiza un "caso exitoso", como es el de la empresa Huawei Technologies. La selección de esta firma obedece a varias razones. Es considerada un ejemplo paradigmático de la temprana creación de firmas tecnológicas privadas en el sur del país, con una posición actual de liderazgo en el mercado mundial de telecomunicaciones fijas y móviles, en redes $5 G$ y en IA. Es considerada un "caso exitoso" de internacionalización de firmas tecnológicas chinas, un "ícono" del renovado poderío tecno-industrial chino y blanco principal de sanciones y prohibiciones impuestas por Estados Unidos en el contexto de la disputa con China.

Para elaborar el texto, tomamos un enfoque analítico-descriptivo basado en el aporte de fuentes primarias y secundarias. Entre las primeras, incluimos planes quinquenales elaborados por el gobierno chino, el Plan "Hecho en China 2025" y documentos sobre futuros desarrollos tecnológicos con horizonte hacia el año 2050 elaborados por la Academia de Ciencias Sociales de China (CAS). Estos documentos aportan consistencia empírica al trabajo. Autores nacionales y extranjeros, incluso chinos, así como informes técnicos, brindan ideas y argumentos aplicables al objeto propuesto de estudio.

\section{El inicio del camino (Dao)}

La especificidad que China reviste en su encumbramiento como potencia tecnológica se define por diversas variables y factores. En primer lugar, se encuentra la decisión política adoptada en los albores de las reformas económicas a fines del siglo XX, respecto a la necesidad de generar renovadas capacidades en el campo científico-tecnológico. Cuando China lanzó su política de "puertas abiertas", el capítulo sobre ciencia y tecnología (C\&T) ocupó el tercer lugar dentro de las denominadas "cuatro modernizaciones" (Xiaoping, 1979). En aquel entonces, la dirigencia política coincidió en que el futuro de China inexorablemente debería fundarse, no sólo en su proyección cultural, financiera y militar, sino fundamentalmente en su poderío tecnológico.

El diagnóstico situacional de aquellos años fue muy claro. La China imperial fue la primera economía del mundo hasta mediados del siglo XIX (Madison, 2007). Su espíritu inventivo dio origen a notables descubrimientos determinantes en procesos de mundialización económica y socialización de ideas, como el papel, imprenta de tipos móviles, instrumentos 
astronómicos de medición, el ábaco, métodos de cálculo, la pólvora, etc. Recuperar esa historia exitosa supondría dotar de fundamentos firmes el proceso de transformación económica estructural. Como resultado, maximizar capacidades opacadas bajo un modelo de economía centralmente planificada devino en prioridad estratégica. 4

En sus orígenes, la construcción de poder tecnológico siguió un sendero similar al de otras economías avanzadas de Asia (Corea del Sur, Japón, Taiwán, Singapur). El activo rol del Estado mediante planes nacionales de mediano y largo plazo, la inversión estatal focalizada en sectores considerados estratégicos, el aliento a la radicación de firmas tecnológicas foráneas, la localización de actividades de investigación y desarrollo (I+D) en Zonas económicas especiales (Z.E.E), el aprendizaje tecnológico, la formación de recursos humanos calificados y el establecimiento de interfases con el sector productivo nacional, formaron parte de un amplio menú de incentivos gubernamentales5. Así China se transformó en una plaza atractiva para la radicación de inversiones (ie) en su mayoría proveniente de economías asiáticas. La deslocalización de industrias mano de obra intensivas desde Taiwán y Hong Kong retroalimentó el proceso de aprendizaje y formación de una mano de obra calificada. Normas y regulaciones nacionales favorecieron el establecimiento de joint ventures (JV) entre firmas nacionales y extranjeras con la condición de transferir tecnologías y conocimientos sobre gestión corporativa. Este "efecto derrame" brindaría el impulso inicial para la creación de empresas tecnológicas -estatales y privadas- gracias a la absorción de know-how en su mayor parte proveniente de Occidente.

En esta primera etapa, el rol del Estado fue determinante en la selección de sectores prioritarios, asignaciones presupuestarias, otorgamiento de incentivos crediticios y fiscales para la radicación de firmas tecnológicas extranjeras, contratación de técnicos y expertos foráneos, construcción de infraestructura educativa, apertura de nuevos centros de investigación, creación de parques tecnológicos y diseño de instrumentos financieros específicos. El clima de negocios resultante posibilitó que, hacia fines del siglo pasado, surgieran empresas tecnológicas hoy íconos de China, como Huawei, fundada en 1987 por Zheng Rengfei en la zona económica especial de Shenzhen (sur del país).

Durante la década del noventa del siglo XX, los gastos de China en I\&D se triplicaron; entre 1991 y 1999 el gasto en C\&T e I\&D como porcentaje del PBI pasó del 1,8 y 0,7\% al 1,57 y 0,83 respectivamente. Las oportunidades creadas por la nueva economía digital afianzaron el sendero establecido generaron condiciones para el despliegue creativo de emprendedores y la ceración de nuevas firmas tecnológicas. Claros ejemplos fueron Alibaba, Tencent, Baidú, ZTE y Lenovo en el sector computacional; el sector aeroespacial afianzó desarrollos satelitales, prioridades sobre cambio en la matriz energética tuvieron como resultado desarrollos en generación nucleoeléctrica, energía fotovoltaica, nuevos materiales, biomedicina para atender necesidades en salud pública, agricultura sostenible, telecomunicaciones, producción de chips y hardware. El entonces zar de la economía, Zhu Rongji, puso en marcha la

4 Bregolat, E. (2014). "La obsesión tecnológica de los nuevos mandarines", en Revista Política Exterior, marzo/ abril, p. 24

5 Naughton, Barry, (Edit.), 1997, The China circle. Economis and technology in the PRC, Taiwan, and Hong Kong, Brooking institutions Press, Washington DC. 
política experimental de gufenhua, con la cual permitía la quiebra o fusión de empresas estatales y su concurrencia al mercado de capitales para financiar operaciones; entre las principales, bancos, aviación, transporte y telecomunicaciones. 6 Producto de lo expuesto, en 1998 China exportó 30.000 millones de dólares en productos de alta tecnología, lejos aún de los Estados Unidos con 190.000 millones de dólares o Japón con 90.000 millones de dólares (Shenkar, 2005).

Los avances en la liberalización económica e internacionalización de empresas (Go out policy) alentó la globalización de firmas tecnológicas chinas. En el contexto de la nueva economía digital, la formación de empresas transnacionales de base tecnológica evidenció la adopción de formatos organizacionales y corporativos en una escala que las habilitó para competir con sus pares de Occidente y Asia. Empresas tecnológicas públicas y privadas, altamente flexibles7, internacionalizaron sus operaciones en sectores de punta, como microelectrónica, biotecnología, nanoelectrónica, robótica, TICs, petroquímica, nuevos materiales, optometría, energías no convencionales, etc. 8 Firmas estadounidenses como Qualcomm, Intel, Broadcom y Texas Instruments (TI) consolidaron operaciones en el continente proveyendo microprocesadores a empresas como Huawei, ZTE y Xiaomi; a ellas se sumaron Apple o Intel en la producción de semiconductores, así como la empresa taiwanesa Foxconn en la producción de equipamiento, con operaciones principalmente desde la Zona Económica Especial de Shenzhen. Favorecidas por un entorno de rápida apertura económica en la búsqueda china por ingresar a la Organización Mundial de Comercio (OMC), las firmas occidentales recibieron a miles de ingenieros chinos en el Silicon Valley y en las Universidades, quienes luego replicarían dichas capacidades en parques tecnológicos específicamente diseñados para financiar start-up industries en sectores tecnológicos de punta.

La concepción planificadora central dio alta prioridad al establecimiento por parte de firmas extranjeras de Centros de I\&D; tales fueron los casos de General Motors, Oracle, Siemens, Nokia, IBM, Hewlett Packard, Toyota, Ford, Volkswagen, entre otras. Filiales estadounidenses ampliaron sus operaciones en China, junto con el compromiso de inversión en I\&D. En el año 2000, China ocuparía la undécima posición en la lista de países receptores de inversiones en I\&D por parte de firmas estadounidenses (decimocuarta en 1994).

Los avances en el desarrollo tecnológico para uso civil nunca estuvieron desligados de sus potenciales aplicaciones en el campo de la defensa y seguridad nacional. Ejemplo paradigmático han sido las redes de telefonía fija y móvil, el descubrimiento de nuevos materiales y los avances en biomedicina militar. Como lo como expresa Wo Lap Lam “bajo la visión de la 'fusión de la paz y la guerra', el general Chi Haotian pidió que el ejército juegue un papel más importante en el transporte civil y las telecomunicaciones. Incluso en tiempos

6 Lam, W. W. L. (1999). The Era of Jiang Zemin. Pearson PTR.

7 López, A y Ramos, D (Coord.), (2010), Las Exportaciones de Servicios en América Latina: Los casos de Argentina, Brasil y México, Serie Red Mercosur.

8 Jonas Nahm y Edward S. Steinfeld, (2013), Scale up Nation: China's specialization in innovative manufacturing, Massachusetts Institute of Technology y Brown University, pp.12-28. 
ordinarios, el desarrollo nacional y regional en el ámbito del transporte y las telecomunicaciones debe tener en cuenta las necesidades de defensa" 9 .

Un último aspecto destaca el liderazgo ejercido por una nueva generación de empresarios; nóveles emprendedores, como Jack Ma (fundador y Presidente Ejecutivo de Alibaba), propiciaron un cambio radical en la concepción y operación de firmas en sectores tecnológicos de punta. Hijos de las reformas, asumieron el desafío de innovar con el solo objetivo de capturar nichos de mercado interno e internacional para sus firmas. La "virtualidad" de los nuevos negocios y su desacople con anclajes geográficos expandió sus capacidades en gran parte adquiridas a imagen y semejanza de sus pares estadounidenses.

A medida que la liberalización del comercio mundial amplificaba opciones de inserción externa e integración en cadenas globales de valor (GVC), las fábricas chinas de semiconductores, equipos de telefonía, electrónica, automotrices, computadoras se transformarían tanto en clientes como proveedores estratégicos para los Estados Unidos, mercados asiáticos y europeos.

\section{Continuidad y reafirmación}

A comienzos del siglo XXI, sectores considerados estratégicos fueron incluidos en sucesivos planes quinquenales. EI XI Plan quinquenal 2006-2010 establecía la formación de grupos regionales con el fin de estimular el crecimiento económico, aumentar la productividad, consolidar cadenas regionales de producción, establecer corredores internos de desarrollo, alentar la innovación y aumentar la productividad en sectores exportadores. Si bien China ya contaba con grupos avanzados en zonas como el Delta del Río Yangtze (centrado en Shanghai) y el Delta del Río Perla (con epicentro en Guangzhou), el plan contemplaba ampliar la dotación mediante una conformación geoespacial en red asentada en diecinueve áreas compuestas por dos ciudades como núcleo vinculadas con nuevos centros urbanos o ciudades satélites.

En un contexto de acelerado crecimiento (11,2\% promedio anual), el XII Plan Quinquenal (2011-2015) priorizó el impulso de siete industrias (SEIS, por sus siglas en inglés, seven strategic emerging industries) consideradas "motores de la economía nacional" para las siguientes décadas: i) tecnologías de energías limpias, ii) TI de nueva generación, iii) biotecnologías, iv) equipamiento de alta complejidad tecnológica, v) energías alternativas, vi) nuevos materiales y vii) vehículos propulsados por energías alternativas. EI XIII Plan Quinquenal (2016-2020), aún vigente, contempla la importancia del desarrollo tecnológico para el crecimiento económico, subraya la necesidad de continuar apostando a la innovación productiva, el desarrollo coordinado y ecológico, y la apertura para generar un cambio estructural en la economía.10

\footnotetext{
9 Lam (1999) ibídem. Op.cit, pág. 167.
}

10 EI XII Plan Quinquenal de la República Popular China, Oficina Económica y Comercial de la Embajada de España en Shanghái. 


\section{Presente y futuro}

El sendero seguido por China le permite ocupar posiciones de liderazgo en sectores tecnológicos de punta y mostrar evidentes logros en innovación. Según la OCDE, el gasto de China en I\&D aumento un 8,6\% en 2018 (8,1\% en 2017); y medido por la paridad de poder de compra (PPP) en términos absolutos para dicho año, China fue el segundo país que más invirtió en I + D, con \$ 468.000 millones de dólares luego de Estados Unidos con \$582.000 mil millones11 (Gráfico 1).

Gráfico 1: Gasto interno bruto en I\&D, 2000 - 2018.

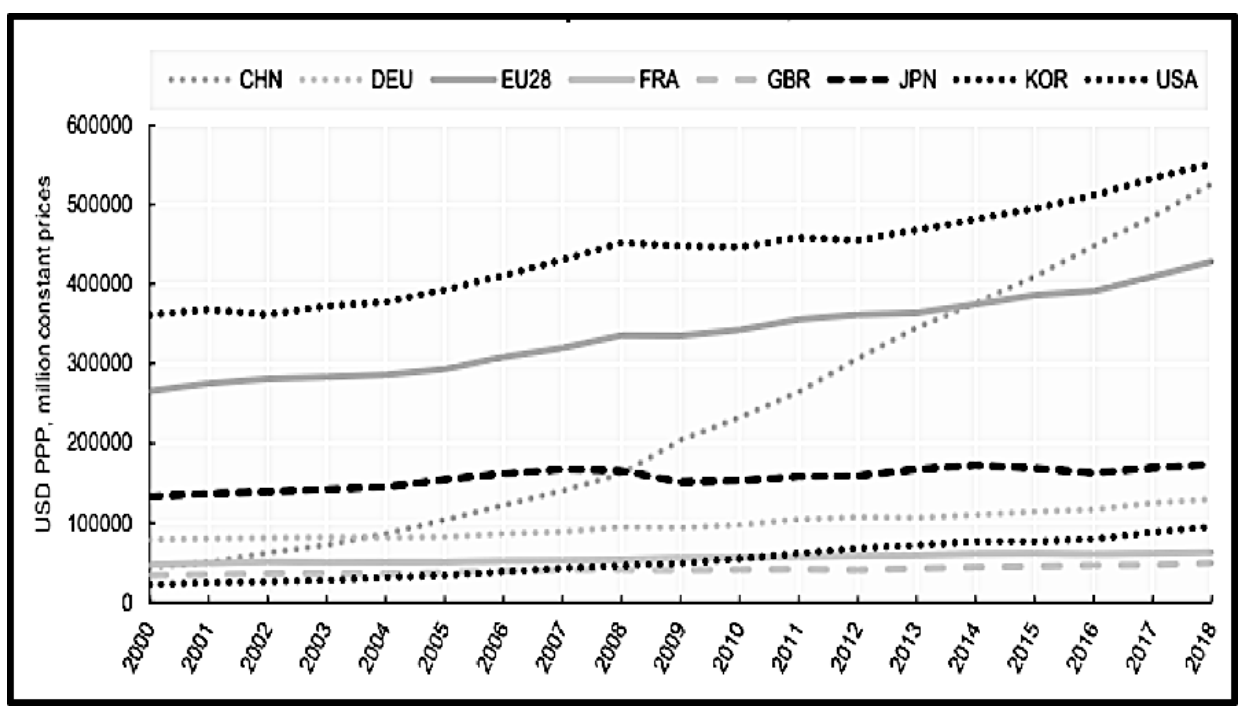

Fuente: OCDE main Science and Technology database, febrero, 2020. http://oe.ced/msti.

De acuerdo a la Oficina Nacional de Estadísticas de China, el gasto total público y privado en ciencia y tecnología en 2019 aumentó un 12,5\% con respecto al año anterior, hasta alcanzar los 322.000 millones de dólares. El gasto en investigación básica representó el $6 \%$ del total;ç, distribuido de la siguiente forma: investigación aplicada, 11,3\%, y desarrollo, $82,7 \%$; equivalente al $2,23 \%$ del PIB. El objetivo sobre gasto en I+D para 2020 fue fijado en 2,5\% del PIB.12

De acuerdo al Global Innovation Index 2019, China ocupó la décimo cuarta posición en 2019 (17 en 2018), pero si se la compara con otras economías de ingreso medio-alto, fue

11 La OCDE en su conjunto gastó el 2,38\% del PIB I\&D durante 2018, Israel y Corea del Sur gastaron el 4,9\% y el $4,5 \%$ del PIB, respectivamente.

12 OECD, Main Science and Technology Indicators R\&D Highlights in the February 2020, en https://www.oecd.org/sti/msti2020.pdf. 
la primera de Asia seguida por Malasia13. Esta progresiva toma de posiciones en nichos tecnológicos antes dominados por firmas extranjeras se verifica con el número de solicitudes de patentes: Huawei Technologies (equipamiento de telecomunicaciones) lidera los registros del Patent Cooperation Treaty (PCT) desde hace un lustro, seguida por Oppo Mobile (móviles y electrónica de consumo), BOE Technology (electrónica, loT, cuidado de la salud), Ping An Technology (plataformas) y ZTE (equipamiento de telecomunicaciones), las que ocupan lugares dentro de las veinte firmas que más solicitudes publicaron durante 2019 (Ver lista).

\section{Listado de las $\mathbf{2 0}$ empresas con más solicitudes anuales de patentes en el PCT (Años 2017, 2018, 2019)}

\begin{tabular}{|c|c|c|c|c|c|c|}
\hline \multirow[b]{2}{*}{ Ranking } & \multirow{2}{*}{$\begin{array}{l}\text { Change in } \\
\text { position } \\
\text { from } 2018\end{array}$} & \multirow[b]{2}{*}{ Applicant } & \multirow[b]{2}{*}{ Origin } & \multicolumn{3}{|c|}{ Published PCT applications } \\
\hline & & & & 2017 & 2018 & 2019 \\
\hline 1 & 0 & HUAWEI TECHNOLOGIES CO., LTD. & China & 4,024 & 5,405 & 4,411 \\
\hline 2 & 0 & MITSUBISHI ELECTRIC CORPORATION & Japan & 2,521 & 2,812 & 2,661 \\
\hline 3 & 2 & SAMSUNG ELECTRONICS CO., LTD. & Republic of Korea & 1,757 & 1,997 & 2,334 \\
\hline 4 & -1 & QUALCOMM INCORPORATED & U.S. & 2,163 & 2,404 & 2,127 \\
\hline 5 & 12 & $\begin{array}{l}\text { GUANG DONG OPPO MOBILE TELECOMMUNICATIONS } \\
\text { CORP., LTD }\end{array}$ & China & 474 & 1,042 & 1,927 \\
\hline 6 & 1 & BOE TECHNOLOGY GROUP CO.,LTD & China & 1,818 & 1,813 & 1,864 \\
\hline 7 & 2 & TELEFONAKTIEBOLAGET LM ERICSSON (PUBL) & Sweden & 1,564 & 1,645 & 1,698 \\
\hline 8 & 53 & PING AN TECHNOLOGY (SHENZHEN) CO., LTD. & China & 23 & 336 & 1,691 \\
\hline 9 & 1 & ROBERT BOSCH CORPORATION & Germany & 1,354 & 1,525 & 1,687 \\
\hline 10 & -2 & LG ELECTRONICS INC. & Republic of Korea & 1,945 & 1,697 & 1,646 \\
\hline 11 & 9 & LG CHEM, LTD. & Republic of Korea & 850 & 969 & 1,624 \\
\hline 12 & 0 & $\begin{array}{l}\text { PANASONIC INTELLECTUAL PROPERTY MANAGEMENT } \\
\text { CO., LTD. }\end{array}$ & Japan & 1,280 & 1,465 & 1,567 \\
\hline 13 & 0 & SONY CORPORATION & Japan & 1,735 & 1,342 & 1,566 \\
\hline 14 & 1 & HEWLETT-PACKARD DEVELOPMENT COMPANY, L.P. & U.S. & 1,519 & 1,170 & 1,507 \\
\hline 15 & -4 & MICROSOFT TECHNOLOGY LICENSING, LLC & U.S. & 1,536 & 1,476 & 1,370 \\
\hline 16 & 5 & FUJIFILM CORPORATION & Japan & 970 & 962 & 1,158 \\
\hline 17 & -3 & SIEMENS AKTIENGESELLSCHAFT & Germany & 1,063 & 1,211 & 1,153 \\
\hline 18 & -14 & ZTE CORPORATION & China & 2,965 & 2,080 & 1,085 \\
\hline 19 & 0 & DENSO CORPORATION & Japan & 968 & 998 & 1,026 \\
\hline 20 & 2 & NEC CORPORATION & Japan & 899 & 947 & 1,024 \\
\hline
\end{tabular}

Fuente: WIPO (2020)

En la lista Global 2000 de Forbes, que incluye las principales firmas tecnológicas a nivel mundial, Apple mantuvo su posición como líder, en tanto Tencent, el conglomerado chino, se encuentra entre las ocho principales compañías del mundo por su valor de mercado14. Si se miden logros por el número de "unicornios" (empresas que ostentan un valor de mercado superior a los 1.000 millones de dólares), acorde a datos de Hurun Global Unicorn Index15, Estados Unidos aún lidera la lista a nivel mundial con 233 sobre 227 de China; entre ambos concentran el $80 \%$ de mayor valor que figuran en la lista. No obstante, China hoy es

13 WIPO, https://www.globalinnovationindex.org/userfiles/file/reportpdf/GII_2019_EN_English.pdf.

14 Ver: https://forbes.co/2020/05/15/tecnologia/las-companias-tecnologicas-mas-grandes-del-mundo2020-apple-se-mantiene-en-la-cima/

15 Ver: https://www.hurun.net/EN/HuList/Unilist?num=ZUDO23612EaU, consultada el 20 de septiembre de 2020. 
el hogar de los tres unicornios más valiosos del mundo.16 Ant Group, compañía fintech afiliada a Alibaba, ocupa el primer lugar por su valor de Mercado (143.000 millones de dólares), Beijing ByteDance Technology, controladora de TikTok, el segundo lugar (80.200 millones de dólares), y la tercera es el gigante de transporte compartido Didi Chuxing.

De acuerdo al estudio, en China los unicornios prevalecen en al menos trece sectores industriales, con mayor componente tecnológico, como servicios empresariales, medios y entretenimiento, transporte y automotriz, y fintech (servicios de tecnología financiera digital). Ejemplos de unicornios en estos sectores son ManBang Group (transporte de camiones), Meicai (una plataforma B2B para la industria agrícola), ByteDance (desarrollador de la plataforma de contenido Toutiao y la popular aplicación de video de 15 segundos TikTok), Yitu (servicios de inteligencia artificial IA a empresas) o Tuhu, que conecta personas con servicios relacionados con el automóvil en línea. Por su localización geográfica, el $80 \%$ de estos unicornios tiene su sede en ciudades como Beijing, Shanghai, Shenzhen o Hangzhou. Para varias de estas empresas, sus planes de expansión global contemplan aprovechar las oportunidades que brindan proyectos asociados a la Iniciativa de la Franja y la Ruta de la Seda (OBOR) 17.

Durante la última Asamblea Legislativa (APN) celebrada en mayo de 2020, el gobierno central reafirmó los planes 2025 y 2050, así como la inversión de 1,4 billones de dólares en un programa de infraestructura digital. Estos buscan desacoplar tecnológicamente a China de los Estados Unidos, enfrentar la creciente competencia global, responder a medidas restrictivas sobre provisión de insumos estadounidenses y/o europeos, y ajustar las condiciones operativas de sus empresas a un mundo post pandemia con cadenas globales (CG) y regionales (CR) fragmentadas bajo determinantes de un nuevo "soberanismo" económico y deterioro del multilateralismo comercial institucionalizado en la crisis de la Organización Mundial de Comercio (OMC). Corolario de las nuevas reglas, las empresas extranjeras deberán adoptar modelos de negocios "en China para China" si desean acceder al mercado interno18.

En su trayectoria como potencia global, el Documento Mapa de Ruta para la Ciencia y Tecnología en el 2050 (2010), elaborado por la Academia China de Ciencias (CAS, en su sigla en inglés), define objetivos en C\&T con el objeto de lograr un modelo como "high-tech economy" para dicho año. Los sectores seleccionados en el documento prospectivo son: sistemas de energías y recursos sustentables, materiales avanzados y manufacturas ecológicamente inteligentes, nuevas redes y sistemas de información (hacia la "sociedad del conocimiento", universalización de las TICs, mayor capacidad de las redes y servicios de informa-

16 Ver: https://www.bworldonline.com/us-china-lead-number-of-unicorns-in-global-unicorn-index-2020/, consultada el 21 de septiembre de 2020.

17 Jianbin Gao and Yuqing Guo, 2019, China's herd of unicorns. About 200 Chinese startups have valuations of $\$ 1$ billion or more, and many are preparing to go global, en. https://www.strategy-business.com/article/Chinas-herd-of-unicorns?gko=5a81d.

18 Ver, Capri, Alex, 2020, "Export controls and the rise of US-China techno-nationalism", link: https://technode.com/author/alexander-capri/. 
ción, sistema de seguridad social en salud, desarrollo de la conservación ecológica y medioambiental, expansión de la capacidad de exploración del espacio y el océano, sistema Nacional de Seguridad Pública con el fin de asegurar el uso pacífico del espacio exterior, transporte oceánico y protección ante amenazas biológicas y ecológicas. En síntesis, hacia el 2050, China aspira aprovechar oportunidades abiertas por cambios transformadores en $\mathrm{TI}$, mejorar habilidades pro innovación, fomentar el desarrollo sostenible y lograr, para la futura sociedad china, un nivel de informatización cercano al de países desarrollados. ${ }^{19}$

\section{El Plan China 2025 y la puja tecnológica con Estados Unidos}

El Plan Hecho en China 2025 es la manifestación concreta de la voluntad nacional por consolidar su status como potencia tecnológica durante el siglo XXI. Lanzado por el presidente Xi Jinping en 2015, busca reducir brechas con Occidente y sostener la competitividad general de la economía. También suele ser visto como una respuesta a los desafíos que enfrenta la industria manufacturera nacional ante la pérdida de competitividad por mayores costos laborales y la consiguiente necesidad de fortalecer bases nacionales de innovación superando obstáculos institucionales disfuncionales.20

Las principales sectores incluidos en el Plan son: IT de nueva generación, maquinaria de alta gama de control numérico, robótica, equipos aeroespaciales, equipos para ingeniería marítima y fabricación de embarcaciones de alta tecnología, equipos ferroviarios avanzados, vehículos eléctricos, equipos eléctricos, maquinaria y equipos agrícolas, nuevos materiales, productos biofarmacéuticos y dispositivos médicos de alto rendimiento. De concretarse las metas, el país debería alcanzar un $40 \%$ de componentes básicos y materiales básicos fabricados en el país para 2020 (70\% en 2025), una reducción de 30\% en costos operativos, ciclos de producción y tasas de defectos en productos para 2020 (50 \% para 2025) y el establecimiento de quince centros de innovación para el año 2020, número que aumentaría hasta cuarenta en 2025.21

19 Yongxiang Lu (Ed. In Chief), Sciences \& Technology in China: A Roadmap to 2050_Science Press, Chinese Academy of Sciences, Beijing 2010

20 China manufacturing 2025, 2017, Putting Industrial Policy Ahead of Market Forces, European Chamber, pág. 8-20

21 Gómez Pérez-Cuadrado, Esther, 2016, Plan Made in China 2025, ICEX, Oficina Económica y Comercial de España en Pekín. 


\section{Los 10 sectores apuntados por el Plan China 2025 para generación de innovación ra- dical}

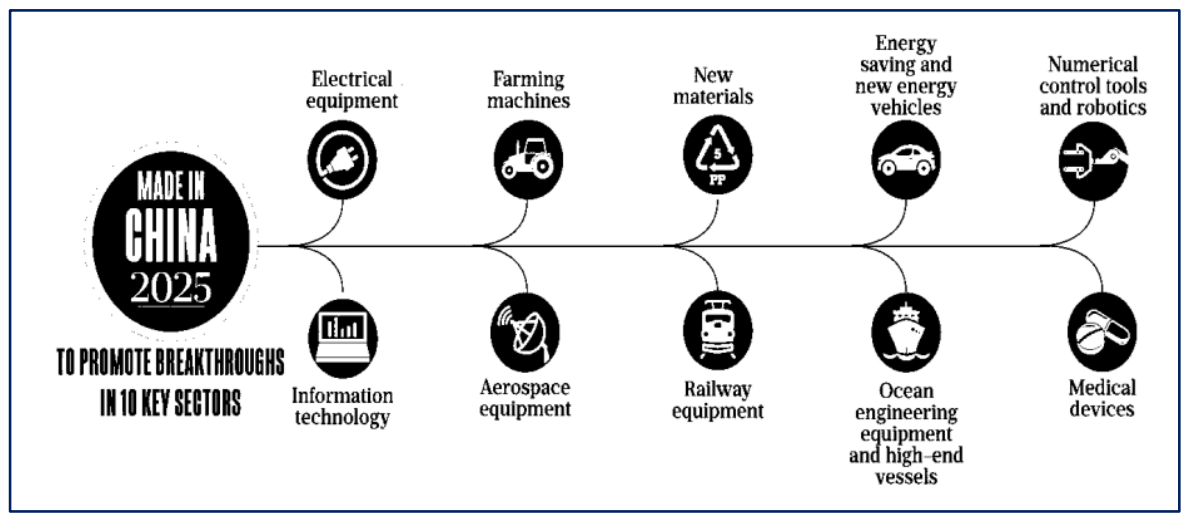

Fuente: HQ Magazine: Revamping “Made in China, 2020

Este plan es el epicentro de la puja competitiva con Estados Unidos. Él estimula el surgimiento de start ups locales que desplacen servicios provistos por compañías extranjeras (principalmente estadounidenses) en áreas particularmente sensibles, como el diseño de microchips. Por este motivo, entre otras metas, el gobierno prevé una inversión de 29.000 millones de dólares destinada a desarrollar una industrial nacional de semiconductores. De esta forma, China aspira ampliar márgenes de "autonomía tecnológica" y reducir su dependencia de proveedores estadounidenses.22 En sintonía con el plan, la creación en 2019 de una versión china del mercado estadounidense de capitales para firmas tecnológicas (Nasdaq), el Star Market busca facilitar el ingreso de capital privado -nacional y/o externo- a startups en dichos sectores. Estas medidas confirman tendencias vinculadas a una paulatina desvinculación entre la primera y segunda economía mundial, una preponderancia en ambas partes de enfoques sesgados hacia el "tecno-nacionalismo" y la presunción compartida sobre escalamiento de hostilidades hoy agudizadas por una frontal guerra comercial.

Los logros de China en el campo tecnológico, sus planes de mediano y largo plazo, y el achicamiento de la brecha de financiación en I\&D respecto de Estados Unidos explican las aprehensiones estadounidenses. Desde el año 2000, según estadísticas provistas por la Fundación Nacional de Ciencias de Estados Unidos (NSF), durante el período comprendido entre 2000 y 2017 , el gasto en I + D en dicho país creció a un promedio de $4,3 \%$ anual, en tanto el

22 Golpe de Estados Unidos a Huawei: corta su acceso a los proveedores mundiales de chips. Huawei continuó utilizando tecnología norteamericana para diseñar semiconductores a pesar de haber sido incluido en una lista negra económica de Washington, David Shepardson, 15 de mayo de 2020. 
registro de China fue de un 17\%. De acuerdo al Informe, de los 2,2 billones de dólares invertidos en I + D a nivel mundial durante 2018, Estados Unidos representó el 25\% y China el $23 \% .23$

La IA y las redes ocupan el centro de las disputas bilaterales, y la competencia abierta entre Estados Unidos y China incluye quién controlará la infraestructura y stándares internacionales en TI. En este contexto, durante 2017 China anunció su ambición de convertirse en líder mundial en IA para 2030. La expectativa de China en aventajar a Estados Unidos en el dominio de IA se sostiene en ventajas tales como el tamaño de su mercado interno y en la ausencia (al menos aparente) de cuestionamientos éticos y sociales que pueden traen aparejadas las aplicaciones de Al a la vigilancia social e individual. Actualmente, China detenta una posición de liderazgo en robótica y dispositivos móviles, ambos grandes usuarios de $\mathrm{Al}$, aún con un gran potencial de expansión doméstica, y además está a la vanguardia en el mercado de aplicaciones para video vigilancia y reconocimiento facial de difundida aplicación en espacios públicos.

En el terreno de 5G, China lidera también la carrera en una nueva generación de tecnologías asociadas a comunicaciones móviles, principalmente en lo concerniente al equipamiento de redes. En el mercado naciente de Quinta Generación existen pocas compañías con plena capacidad para desplegar redes; entre ellas, y las únicas firmas que dominan la nueva arquitectura, son empresas como Huawei y ZTE, la compañía finlandesa Nokia, la sueca Ericsson más el gigante surcoreano Samsung. En todos los casos, las compañías detentan antecedentes determinantes en anteriores desarrollos de standares $2 \mathrm{G}$, $3 \mathrm{G}$ y $4 \mathrm{G} / \mathrm{LTE}$ empleadas en comunicaciones móviles.

Sumado a estos factores, en 2018 cuando la tecnología china 5G comenzó a crecer como alternativa en economías en desarrollo, el Departamento de Estándares Industriales del Comité Nacional de Normalización del país encargó un estudio prospectivo denominado China Standards 2035. Este propone una hoja de ruta estratégica para industrias de tecnología de la información: inteligencia artificial, big data, computación en la nube, 5G y biotecnología. 24 Su objetivo central consiste en respaldar y aprovechar la sólida posición de empresas chinas en mercados emergentes para establecer estándares a favor su favor. En este contexto, América Latina es un importante campo de batalla en el que empresas chinas buscan utilizar su posición en industrias tecnológicas como un vehículo para establecer normas y dominar mercados globales. El empleo industrial eficiente de tecnologías depende de la calidad e interoperabilidad con diferentes sistemas; por lo tanto, los estándares son esenciales al proporcionar una línea de base universal para los múltiples usos y aplicaciones. 25

23 The State of U.S. Science and Engineering 2020 (NSF), 2020, ver https://ncses.nsf.gov/pubs/nsb20201/global-r-d.

24 Horizon Advisory, China Standards series. First report. China Standards 2035, en file://C:/Users/pcescritorio/Documents/Horizon\%20Advisory\%20_\%20Actionable\%20Geopolitical\%20In-

sight\%20_\%20China\%20Standards\%202035.html.

25 China Standards 2035 and the Plan for World Domination-Don't Believe China's Hype, Council on Foreign relations, Blog Post by Guest, Blogger for Net Politics, June 3, 202, en 


\section{Huawei: ¿caso exitoso, ícono tecnológico o amenaza para la seguridad de Europa y Estados Unidos?}

En el marco de la guerra comercial con China, las aprehensiones estadounidenses sobre las operaciones globales de Huawei incluyen desafíos sobre defensa, ciberseguridad y pérdida de mercados por parte de sus firmas tecnológicas. China aventaja a Estados Unidos en desarrollo de redes $5 \mathrm{G}$. Las empresas chinas detentan, además, el 32\% del total de patentes esenciales en tecnología 5G: 16\% en manos de Huawei, 10\% pertenecientes a ZTE y un $3 \%$ en poder de la China Academy of Telecommunications Technology (CATT, Datang Telecom) (Polham, 2019).

En el contexto de una estrategia selectiva de sustitución de importaciones, el crecimiento de la firma tuvo su primer impulso en la protección gubernamental ante la competencia externa. Posteriormente fue beneficiada con créditos otorgados por bancos nacionales destinados a sostener su expansión global. En 2004 logró el apoyo financiero del China Development Bank (CDB) para operaciones de comercio exterior. Huawei cerró entonces contratos de venta de equipos y servicios, con grandes operadoras telefónicas, como Vodafone (Gran Bretaña), Bell Canadá y Telus (Canadá), Vivo (Brasil), Telecom Italia y TATA (India), entre otras. Actualmente, su modelo operativo se sostiene en la reinversión de utilidades en I\&D del orden de un $10 \%$ promedio de sus ingresos por ventas (Gráfico 2). Esto se ha visto reflejado en la apertura de numerosos centros de investigación en China y la deslocalización de operaciones con el fin de sumar capacidades en nuevas tecnologías hacia mercados como India, Suecia y Estados Unidos, entre otros países.

Grafico Nro. 2. Huawei. Gastos en Investigación y Desarrollo sobre Ingresos por Ventas (período 2008-2017)

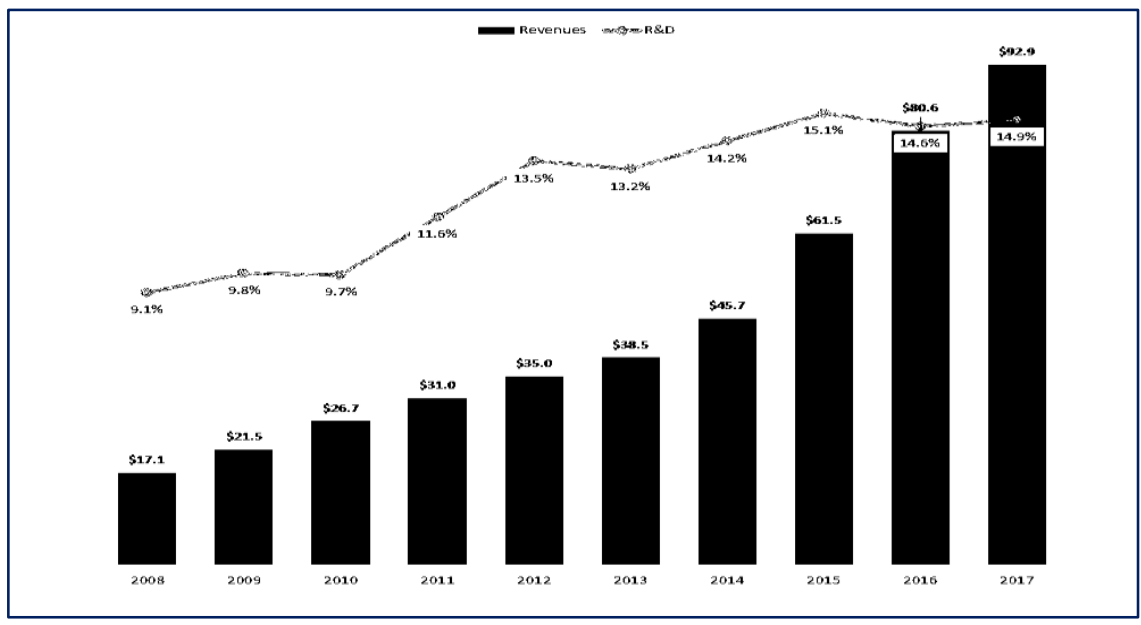

https://www.cfr.org/blog/china-standards-2035-and-plan-world-domination-dont-believe-chinashype 
Fuente: Counterpoint Research (2018)

Ya entrado el siglo XXI, los resultados de la inversión en I+D son verificables en el crecimiento exponencial de solicitudes sobre patentes propias (IPRs). Otro hito que consolidó su posición como líder en el sector 5G fue la adopción en 2017 del Polar Code como estándar de comunicaciones RAN (Radio Access Network), hecho que alarmó aún más a los Estados Unidos ante la amenaza real que constituía al -hasta ese momento- indiscutido liderazgo sectorial a nivel internacional. ${ }^{26}$

Huawei compite en el mercado mundial de equipos de telecomunicaciones y electrónica de consumo, presta servicios asociados y brinda soluciones relacionadas a servicios wireless, inteligencia artificial (AI), cloud, big data, Internet de las Cosas (IoT) y otros servicios de alta tecnología destinados a individuos, gobiernos y/o empresas. Su modelo de negocios, estructura societaria propiedad de Ren Zhengfei más los trabajadores, la pasada pertenencia de su fundador al Ejército Popular de Liberación (EPL) y el tácito apoyo que recibe (subsidios, financiamiento preferencial, compras gubernamentales, etc) por parte del Partido Comunista Chino (PCC) constituyen la principal fuente de sospechas estadounidenses sobre los verdaderos alcances de sus operaciones.

Por tales motivos, las réplicas estadounidenses adoptan variadas formas. El gobierno de Trump lanzó en febrero de 2019 la denominada American Al Iniciative con el fin de mantener el predominio estadounidense en dicho campo, aumentar recursos aplicados a I+D y redireccionar gran parte de sus resultados hacia operaciones anti piratería, cyberseguridad y defensa nacional.27 Mediante un conjunto de normas, regulaciones, prohibiciones y restricciones impuestas por el Ejecutivo y agencias gubernamentales (Departamento de Comercio, Departamento de Justicia), se "deneór el acceso" de firmas chinas a redes nacionales de uso civil y/o militar. Tal es el caso de Huawei o plataformas de entretenimiento, como Tik tok o wechat.

Los argumentos estadounidenses legitiman las medidas como represalia ante constantes ataques de hackers y piratas informáticos chinos que ponen riesgo la seguridad nacional. Estados Unidos también argumenta que la Ley de Inteligencia de China (2017) especifica que las organizaciones deben apoyar, cooperar y colaborar en el trabajo de inteligencia nacional, lo cual significa transferir datos e información obtenida en el exterior al Gobierno Central. La escalada bilateral incluyó el arresto en Canadá, en diciembre de 2018, de Meng Wanzhou, Directora financiera de Huawei, acusada de supuesta violación de sanciones impuestas por Estados Unidos a Irán.

Los desafíos sobre defensa y seguridad aducidos por Estados Unidos, presionan también a miembros de la OTAN. En tal sentido, Gran Bretaña informó que, a partir de fines de 2020, dejará de comprar equipos 5G provistos por Huawei y concedió plazo hasta 2027 para retirar todos los productos de la compañía que componen la infraestructura de red de nueva

26 Polar Codes for Cloud RAN Applications, White Paper Polar Codes for Cloud RAN Applications, Polaran LTD. Agosto de 2018, en: http://www.polaran.com/documents/White_Paper_Polar_Codes_For_CRAN_Applications.pdf.

27 Al Policy- United States. Future of Life Institute https://futureoflife.org/ai-policy-united-states/?cn-reloaded=1\&cn-reloaded=1 (consultado el 27 de julio de 2020) 
generación del país. Otros países han seguido su ejemplo, como Canadá, Australia, Japón y Nueva Zelanda, los que han vetado la compra de equipos y servicios $5 \mathrm{G}$ provistos por la tecnológica china. En el plano de la competencia con tecnológicas estadounidenses, en marzo de 2019, Google canceló la licencia de Huawei para Android, el sistema operativo utilizado en la mayoría de teléfonos móviles en uso a nivel mundial. No obstante los obstáculos, en ALC, Huawei mantiene expectativas por expandir su presencia en Brasil, Argentina, Venezuela y Chile. En el caso de México, según el CEO de América Móvil Carlos Slim, pese al veto estadounidense, la empresa podría continuar empleando equipos chinos provistos por Huawei.

\section{Conclusiones}

Los planes nacionales de China apuntan a posicionarla como potencia tecnológica a mediados del siglo XXI. Se espera que el poderío tecnológico chino mantenga y mejore capacidades competitivas a partir del apoyo estatal, el financiamiento obtenible gracias a la liquidez de sus instituciones financieras y su eficiente canalización hacia empresas medianas y transnacionales. China aspira a ser líder mundial en IA para 2030, lo cual acelera la competencia estratégica con Estados Unidos e impone a la superpotencia global nuevos desafíos en defensa, seguridad y liderazgo tecnológico, extensibles a sus aliados europeos.

El actual contexto de disputas aparenta tener sus límites en la interdependencia entre la primera y la segunda economía del mundo, hoy sujeta a discusión. No obstante, el arte de esta guerra tecnológica puede esconder tanto gruesos trazos como sutiles pinceladas que relativicen esas relaciones. En el caso del gigante asiático, estas líneas parecen estar estudiadas y direccionadas hacia un anhelo de liderazgo mundial que coincida temporalmente con el centenario de la revolución de Nueva China.

\section{Bibliografía}

Bregolat, E. (2014). La obsesión tecnológica de los nuevos mandarines. Revista Política Exterior, marzo/ abril, pp.24

Capri, A. (24 de abril de 2020), Export controls and the rise of US-China techno-nationalism. Technode, Recuperado el 20/09/2020 de https://technode.com/author/alexander-capri/

Deng X. (1987) Problemas fundamentales de la China de hoy, Ediciones de Lenguas extranjeras, Beijing.

Dussel Peters, E. (2014). La inversión extranjera directa de China en América Latina: 10 estudios de caso. Universidad Nacional Autónoma de México.

European Union Chamber of Commerce in China. (2017). China Manufacturing 2025: Putting Industrial Policy Ahead of Market Forces.

Gómez Pérez-Cuadrado, E. (2016). Plan Made in China 2025. ICEX

Harwit, E. (2008), China's telecommunications revolution, Oxford University Press. 
Jorgensen, B. (15 de mayo de 2020) U.S. Moves to Cut Huawei's Access to Global Chip Suppliers, EPSNews

Katz, J. (2015). La macro-y la microeconomía del crecimiento basado en los recursos naturales. Neoestructuralismo y corrientes heterodoxas en América Latina y el Caribe a inicios del siglo XXI. Santiago: CEPAL, 2015. LC/G. 2633-P/Rev. 1. p. 243-259.

Kosacoff, B., López, A., \& Pedrazzoli, M. (2007). Comercio, inversión y fragmentación del mercado global:¿ está quedando atrás América Latina?. Serie Estudios y Perspectivas, 39. CEPAL.

Li, G. (Ed.). (2011). Information Science \& Technology in China: A Roadmap to 2050. Science Press.

Lu, Y. (2009). Science \& technology in China: a roadmap to 2050: Strategic General Report of the Chinese Academy of Sciences. Springer Science \& Business Media.

Maddison, A. (2007). Chinese Economic Performance in the Long Run-Second Edition, Revised and Updated: 960-2030 AD. Paris, FR: OECD Development Centre Studies.

Moneta, C., \& Cesarín, S. (2012). Tejiendo redes. Estrategias de las empresas transnacionales asiáticas en América Latina. EDUNTREF, Argentina.

Nahm, J. and Steinfeld, E.S. (2014). Scale-up nation: China's specialization in innovative manufacturing. World Development, 54, pp.288-300.

National Sciences Foundation (2020), The State of U.S. Science and Engineering 2020. Science \& Engineering Indicators recuperado el 5/09/2020 de https://ncses.nsf.gov/pubs/nsb20201/global-r-d.

Naughton, B. (1995) Growing out of the plan. Chine economic reform 1978-1993, Cambridge University Press.

Naughton, B. (1997) The China circle. Economy and technology in the PRC, Taiwan, and Hong Kong, The Brooking Institution.

Polhman, T. (2019), Who is leading the $5 G$ patent race? A patent landscape analysis on declared 5 G patents and $5 G$ standards contributions, Iplytics Noviembre 2019, https://www.iplytics.com/wp-content/uploads/2019/01/Who-Leads-the-5GPatent-Race_2019.pdf

Rosales, O. (2018). Las claves del conflicto económico China-Estados Unidos. Santiago, Chile: Facultad Latinoamericana de Ciencias Sociales (FLACSO). Recuperado de: http://www. flacsochile. org/slider/las-claves-del-conflicto-economico-chinaestadosunidos.

Shaolong, Y. (2016), The Huawei Way: Lessons from an International Tech Giant on Driving Growth by Focusing on Never-Ending Innovation, McGraw Hill Professional.

Shenkar, O. (2005) El siglo de China; Grupo editorial Norma, Bogotá.

World Intellectual Property Organization WIPO (28 de septiembre de 2020), 
https://www.wipo.int/ipstats/en/statistics/country_profile/profile.jsp?code $=\mathrm{CN}$.

Zheng, Y. (1999). The Outlook for US-China Relations Following the 1997-1998 Summits: Chinese and American Perspectives on Security, Trade, and Cultural Exchange. Chinese University Press. 